\title{
A novel approach of treating gingival recession by Vestibular Incision Subperiosteal Tunnel Access along with palatal connective tissue graft
}

\author{
Surupa Dutta ${ }^{1}$, Farha Nasim ${ }^{2}$, Debarghya Pal ${ }^{3}$, Manoj Kumar Singh ${ }^{4}$, Himadri Chakrabarty ${ }^{5}$ \\ ${ }^{1} 3^{\text {rd }}$ Year Postgraduate, ${ }^{2,3} 2^{\text {nd }}$ Year Postgraduate, ${ }^{4,5}$ Professor, Dept. of Periodontics, Guru Nanak Institute of Dental Sciences \& Research, \\ Kolkata, West Bengal, India \\ *Corresponding Author: Surupa Dutta \\ Email: surupa.mukta@gmail.com
}

\begin{abstract}
Gingival recession, defined as the apical migration of gingival margin possesses great degree of functional and aesthetic problems. Recently new techniques for root coverage have been proposed for treating multiple teeth recession. Keeping in mind the limitations of the conventional techniques, a minimally invasive approach for the recipient site in the aesthetic zone, Vestibular Incision Subperiosteal Tunnel Access (VISTA) technique had been introduced. Since the connective tissue graft is the proven gold standard, it is combined with the minimally invasive technique to achieve the best possible result. Here, a series of such cases are discussed and the 6 months post operative follow up is hence analysed to assess the root coverage.
\end{abstract}

Keywords: Gingival recession, Minimally invasive, Root coverage, VISTA, Connective tissue graft.

\section{Introduction}

Gingival recession is a common feature affecting the population leading to functional and esthetic problems. It is defined as the apical migration of the gingival margin below the cemento-enamel junction (CEJ), leading to root exposure. ${ }^{1}$ Etiological factors such as faulty tooth brushing, malalignment of teeth, periodontal disease, high frenal attachment, traumatic occlusion and iatrogenic factors related to restorative and periodontal treatment procedures are the possible cause for the development of gingival recession. ${ }^{2}$ It is a cause of concern for both the clinician and the patients as it may predispose to further attachment loss, dentinal hypersensitivity and root caries and also appears to be esthetically unpleasant. ${ }^{3}$ An array of treatment modalities are available for the treatment with the objective of root coverage. But most of these are suitable to achieve predictable results in isolated defects. ${ }^{4}$ While gingival recessions frequently affect group of adjacent teeth, therefore, in order to minimize the surgeries and to optimize the esthetic result, all the contiguous recessions should be treated simultaneously. ${ }^{5}$ However, the treatment of multiple recession defects possess great challenge to the clinician owing to extensive avascular root surface area. Other factors like thin gingival phenotype, inadequate keratinized tissue, root prominence and root proximity creates dilemma in the choice of different surgical treatment modalities which further increases the risk of unpredictable outcome.

Although, connective tissue graft (CTG) along with coronally advanced flap was considered to be the gold standard for the treatment due to its high predictability of the treatment outcomes, yet we are looking for an alternative owing to the complications CTG harvesting. ${ }^{6}$ With the introduction of minimally invasive, tunnelling techniques for gingival augmentation, comparable results could be obtained. It attempts to preserve the interdental papillae, providing unhampered blood supply, and faster wound healing. However, these procedures are quite technique sensitive and may cause tissue trauma to sulcular epithelium, leading to unfavourable healing outcomes. ${ }^{4}$ To overcome such complications, the vestibular incision subperiosteal tunnel access (VISTA) approach was introduced by Zadeh.

Further the use of CTG would benefit with faster healing, lesser soft tissue contraction and also increase width and thickness of the keratinized tissue, resulting in better root coverage over a long period of time. ${ }^{7}$ The purpose of this case series is to evaluate clinically, the efficacy of the novel and minimally invasive VISTA in combination with CTG in the treatment of multiple gingival recession defects.

\section{Materials and Methods}

The study was conducted in the Department of Periodontics in Guru Nanak Institute of Dental Sciences and Research, over a period of 6 months. Three patients were selected with gingival recession in mandibular anterior teeth, with the chief complaint of sensitivity in the same region, along with esthetic concern.

\section{Case 1}

A 45 year old female patient with Miller's class III gingival recession in tooth number 41. It was associated with occlusal discrepancy causing interdental bone loss between 31 and 41. Tension test was positive. For treating such a condition, boosting the gingival thickness with connective tissue was adopted in an attempt to cover such gingival recession.

\section{Case 2}

A 32 year old male patient with Miller's class I gingival recession in 31 and 41 had the chief complain of sensitivity of teeth. Minimally invasive VISTA technique for root coverage was adopted, considering the aesthetic zone of concern. 


\section{Case 3}

A 52 year old male patient with Miller's class II gingival recession in 41. Traumatic bite was present in this tooth. Gingival phenotype needed to be boosted owing to the progressing disease condition.

\section{Surgical technique}

The surgical sites were anaesthetized and the recipient sites were prepared by thorough scaling and root planing. The approach for VISTA began with vestibular access incision placed distal to the recession defect as proposed by Zadeh. ${ }^{4}$ The incision was made passing through the periosteum so as to elevate a subperiosteal tunnel, exposing the bone and root dehiscence (Fig. 1). The tunnel was extended to the adjacent teeth beyond the teeth to receive root coverage, to facilitate the mobilization and coronal repositioning of the gingiva. Additionally, the subperiosteal tunnel was extended interproximally under each papilla without making any surface incisions through the papilla, and also below the mucogingival junction to allow low-tension coronal repositioning of the flap. ${ }^{4}$

Palate was chosen as the donor site for obtaining connective tissue graft. The length of the graft is determined by the combined width of the teeth to be covered. The connective tissue graft was harvested using double incision technique (Fig. 2), de-epithelialised (Fig. 3) and then placed inside the tunnel and guided using lasso suturing technique from the other end (Fig. 4). Once properly positioned, the mucogingival complex are then coronally advanced and stabilised using coronally anchored suturing position secured with composite stops (Fig. 5), to prevent apical relapse of the gingival margin during initial stages of healing. ${ }^{4}$ The vertical incision were approximated and sutured.

Root coverage for all 3 patients was done using the same surgical technique. For each patient, antibiotics and analgesic were prescribed and instructed to follow strict oral hygiene maintenance. Sutures at the access incision may be removed after 1 week. Coronally anchored bonded sutures are typically removed at the 3 -week postoperative visit.

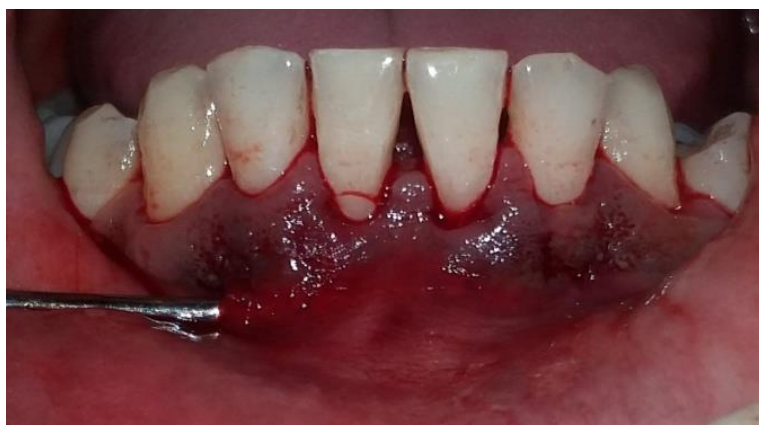

Fig. 1: Vestibular access incision and tunnel preparation

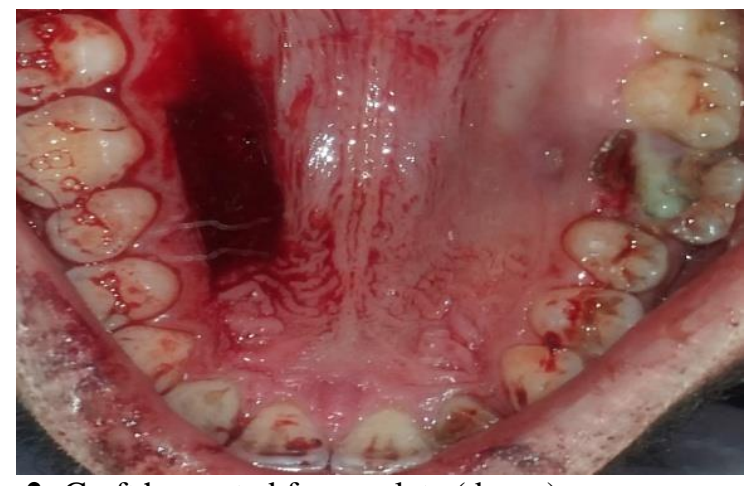

Fig. 2: Graft harvested from palate (donor)

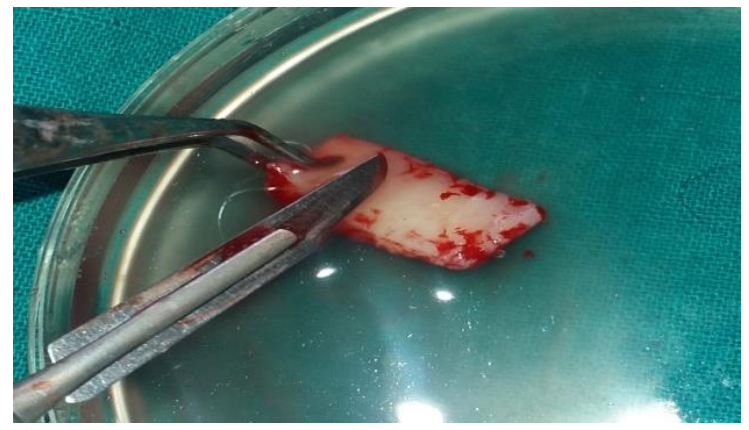

Fig. 3: De-epithelialisation of the graft

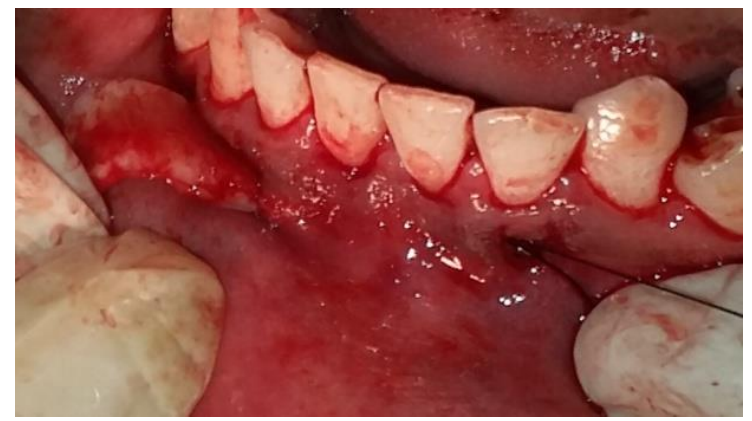

Fig. 4: Graft being guided inside the tunnel using lasso suturing technique

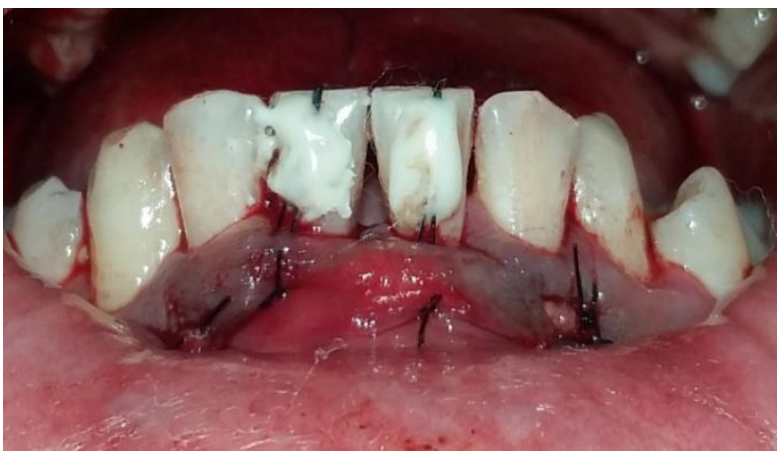

Fig. 5: Coronally anchored suture with composite stops

\section{Result}

Gingival phenotype was appreciably boosted in all the three cases. Owing to the differences in the tissue condition, the result obtained varied considerably (Fig. 6-8). 


\section{Case 1}
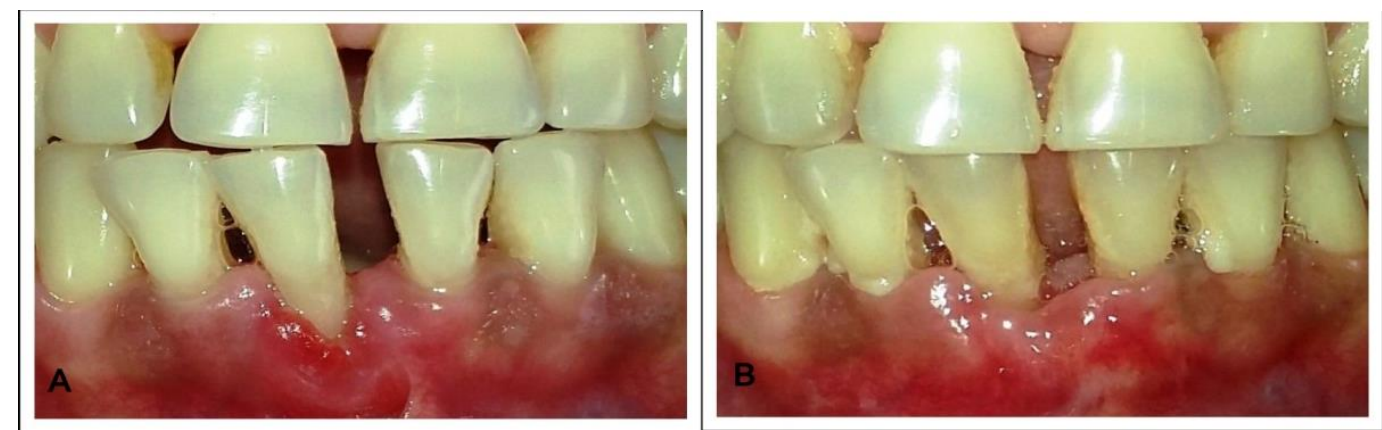

Figure 6: (A): Pre-operative and (B): Post-operative

Case 2
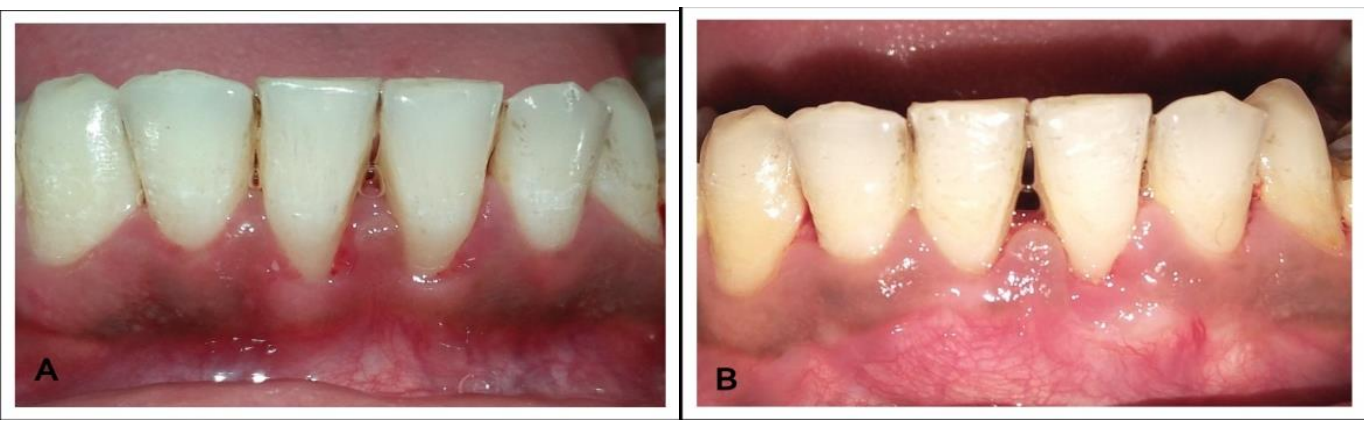

Fig. 7: (A): Pre-operative and (B): Post-operative

\section{Case 3}
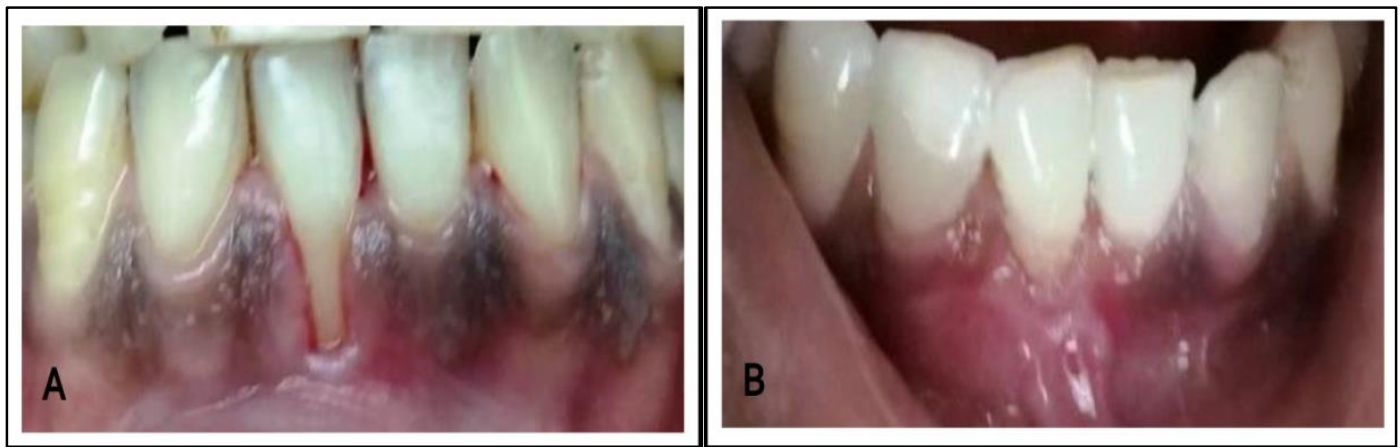

Fig. 8: (A): Pre-operative and (B): Post-operative

\section{Discussion}

With the increasing demand for esthetics in periodontal clinical practice, periodontal plastic surgery aims at treating gingival deformities together with improving esthetics with less scarring and appropriate blending of tissue colour \& texture post-surgery. The minimally invasive tunnelling technique was proposed to preserve esthetics, avoid relapse of gingival augmentation and preserve integrity of interdental papilla. However, this procedure is quite technique-sensitive and cause increased risk of traumatizing and perforating sulcular epithelium, which may result in unfavourable outcomes. ${ }^{4}$

Keeping these limitation in mind, the approach of vestibular incision subperiosteal tunnel access (VISTA) was developed by Zadeh, wherein a vertical incision is placed in the vestibule and a subperiosteal tunnel is created sufficiently beyond the mucogingival margin and through the gingival sulci, facilitating low-tension coronal repositioning of the gingiva while at the same time maintaining the anatomical integrity of the interdental papillae by avoiding papillary reflection. The vestibular incision reduces the possibility of traumatizing the gingiva of the teeth being treated. ${ }^{8}$ Moreover, the subperiosteal dissection through the vestibular incision reduces muscle pull during healing, preventing the relapse of the treated recession. ${ }^{4}$ The vertical incision ensures less disruption of the collateral blood supply, favouring healing at the surgical site. $^{4}$

One of the most important factors increasing the risk for relapse of gingival recession may be thin and delicate 
marginal tissue, therefore, an increased thickness of the keratinized tissue should also be taken care of. ${ }^{9}$ In this case, additional grafting with CTG provides a scaffold to support wound healing by increasing the thickness of the wound area which would compensate for the apical migration of the gingival margin during the late stages of wound healing. Thick gingival tissues has shown greater resistance to recession due to surgical trauma and tissue remodelling following different surgical procedures. ${ }^{10}$

The gingival margin was advanced to the most coronal level of the adjacent interproximal papillae and stabilized with coronally anchored suturing, so as to promote healing by preventing micromotion of the tissue, which is critical for better healing as any micromotion between the graft and the surgical site would impair the collateral circulation to the graft. ${ }^{4}$

The VISTA approach introduced by Zadeh, in an attempt to overcome the shortcomings of the intrasulcular tunnelling technique, offered predictable root coverage in the esthetic zone, with less scarring and post-operative complications as was seen with other techniques. With this technique predictable root coverage and increased width of keratinised tissue was obtained and maintained over a longer time period.

The clinical cases presented in this report had shown an appreciable amount of root coverage with an increase in the width as well as the thickness of keratinised tissue over a period of 6 months.

\section{Conclusion}

VISTA was chosen as a treatment option owing to its advantages over other techniques. Clearly, long-term follow-up with clinical and histological studies are required to obtain more information about the CTG-reinforced VISTA technique for their root coverage and to obtain the predictability of this technique.

\section{Source of funding}

None.

\section{Conflict of interest}

None.

\section{References}

1. Chambrone L, Sukekava F, Araujo MG, Pustiglioni FE, Chambrone LA, Lima LA. Root coverage procedures for the treatment of localised recession-type defects. Cochrane Database Syst Rev 2009;2:CD007161

2. Kassab MM, Cohen RE. The etiology and prevalence of gingival recession. J Am Dent Assoc 2003;134:220-5.

3. Oates TW, Robinson M, Gunsolley JC. Surgical therapies for the treatment of gingival recession. A systematic review. Ann Periodontol 2003;8:303-20.

4. Zadeh HH. Minimally invasive treatment of maxillary anterior gingival recession defects by vestibular incision subperiosteal tunnel access and platelet-derived growth factor BB. Int $J$ Periodontics Restor Dent 2011;31:653-60.

5. Zucchelli G, De Sanctis M. Treatment of multiple recessiontype defects in patients with esthetic demands. $J$ Periodontol 2000;71:1506-14.
6. Cairo F, Pagliario U. Treatment of gingival recession with coronally advanced flap procedures. A systematic review. $J$ Clin Periodontol 2008;35:136-62.

7. Cortellini P, Tonetti M, Baldi C, Francetti L, Rasperini G, Rotundo R. Does placement of a connective tissue graft improved the outcomes of coronally advanced flap for coverage of single gingival recession in upper anterior teeth? A multi-centre, randomized, double-blind, clinical trial. J Clin Periodontol 2009;36:68-79.

8. Reddy S. Vestibular incision subperiosteal tunnel access (VISTA) with platelet rich fibrin (PRF) and connective tissue graft (CTG) in the management of multiple gingival recessionA case series. Int J Appl Dent 2016;2:34-7.

9. Müller HP, Eger T. Masticatory mucosa and periodontal phenotype: A review. Int J Periodontics Restor Dent 2002;22:172-83.

10. Trombelli L, Simonelli A, Minenna L, Rasperini G, Farina R. "Effect of a connective tissue graft in combination with a single flap approach in the regenerative treatment of intraosseous defects. J Periodontol 2017;88:348-56.

How to cite: Dutta S, Nasim F, Pal D, Singh MK, Chakrabarty H. A novel approach of treating gingival recession by Vestibular Incision Subperiosteal Tunnel Access along with palatal connective tissue graft. IP Int J Periodontol Implantol 2020;5(1):37-40. 